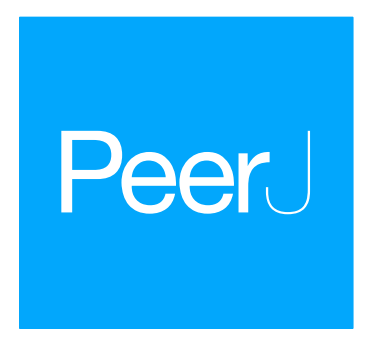

\title{
Extracorporeal life support with left ventricular decompression-improved survival in severe cardiogenic shock: results from a retrospective study
}

\author{
Bastian Schmack ${ }^{1, *}$, Philipp Seppelt ${ }^{1,2, *}$, Alexander Weymann ${ }^{3}$, Christina Alt ${ }^{1}$, \\ Mina Farag ${ }^{1}$, Rawa Arif ${ }^{1}$, Andreas O. Doesch ${ }^{4}$, Philip W. Raake ${ }^{4}$, \\ Klaus Kallenbach ${ }^{5}$, Ashham Mansur ${ }^{6}$, Aron-Frederik Popov ${ }^{7}$, Matthias Karck ${ }^{1}$ \\ and Arjang Ruhparwar ${ }^{1}$ \\ ${ }^{1}$ Department of Cardiac Surgery, University Hospital Heidelberg, Heidelberg, Germany \\ ${ }^{2}$ Medical Clinic III, Department of Cardiology, University Hospital Frankfurt, Frankfurt, Germany \\ ${ }^{3}$ Department of Cardiac Surgery, University Hospital Oldenburg, Oldenburg, Germany \\ ${ }^{4}$ Department of Cardiology, University Hospital Heidelberg, Heidelberg, Germany \\ ${ }^{5}$ Department of Cardiac Surgery, INCCI National Heart Institute, Luxembourg, Luxembourg \\ ${ }^{6}$ Department of Anesthesiology, Emergency and Intensive Care Medicine, University Medical Center, \\ University of Goettingen, Goettingen, Germany \\ ${ }^{7}$ Division of Thoracic and Cardiovascular Surgery, University Hospital Frankfurt, Frankfurt, Germany \\ These authors contributed equally to this work.
}

Submitted 10 July 2017 Accepted 25 August 2017 Published 29 September 2017

Corresponding author

Bastian Schmack,

bastian.schmack@med.uni-

heidelberg.de

Academic editor

Mandeep Mehra

Additional Information and Declarations can be found on page 12

DOI 10.7717/peerj.3813

Copyright

2017 Schmack et al.

Distributed under Creative Commons CC-BY 4.0

OPEN ACCESS

\section{ABSTRACT}

Objective. Extracorporeal life support (ECLS) is a life-saving procedure used in the treatment of severe cardiogenic shock. Within this retrospective single centre study, we examined our experience in this critically ill patient cohort to assess outcomes and clinical parameters by comparison of ECLS with or without selective left ventricular decompression.

Methods. Between 2004 and 2014 we evaluated 48 adult patients with INTERMACS level 1 heart failure (age $49.7 \pm 19.5$ years), who received either central ECLS with ( $n=20,41.7 \%)$ or ECLS without $(n=28,58.3 \%$, including 10 peripheral ECLS) integrated left ventricular vent in our retrospective single centre trial.

Results. Follow up was $100 \%$ with a mean of $0.83 \pm 1.85$ years. Bridge to ventricular assist device was feasible in $29.2 \%(n=14)$, bridge to transplant in $10.4 \%(n=$ $5)$ and bridge to recovery in $8.3 \%(n=4)$. Overall 30 -day survival was $37.5 \%$, 6-month survival $27.1 \%$ and 1-year survival $25.0 \%$. ECLS support with left ventricular decompression showed favourable 30-day survival compared to ECLS without left ventricular decompression $(p=0.034)$. Thirty-day as well as long-term survival did not differ between the subgroups (central ECLS with vent, ECLS without vent and peripheral ECLS without vent). Multivariate logistic regression adjusted for age and gender revealed ECLS without vent as independent factor influencing 30-day survival. Conclusion. ECLS is an established therapy for patients in severe cardiogenic shock. Independent of the ECLS approach, 30-day mortality is still high but with superior 30-day survival for patients with ECLS and left ventricular venting. Moreover, by unloading the ventricle, left ventricular decompression may provide an important time window for recovery or further treatment, such as bridge to bridge or bridge to transplant. 
Subjects Cardiology, Clinical Trials, Emergency and Critical Care, Surgery and Surgical Specialties

Keywords Acute heart failure, Cardiogenic shock, Extracorporeal circulation, ECLS, ECMO

\section{INTRODUCTION}

Cardiogenic shock following acute or chronic heart failure is still associated with poor overall survival (Awad et al., 2012; Combes et al., 2008; Hochman et al., 1995; TRIUMPH Investigators et al., 2007). For critically ill patients with INTERMACS (Interagency Registry for Mechanically Assisted Circulatory Support) level 1 ("critical cardiogenic shock") the use of veno-arterial extracorporeal life support (ECLS) is often the last therapeutic option for maintaining circulation and providing a longer window of time for further treatment. ECLS has proved its therapeutic value both during on-going cardio-pulmonary resuscitation and in the period immediately after successful cardio-pulmonary resuscitation, when end-organ function is severely impaired.

Patients with severe cardiogenic shock often present with both left and right heart failure. Low-output LV failure causes concomitant insufficient organ perfusion, while inadequate decompression of the failing left ventricle (LV) causes a backlog of blood into the lung with subsequent pulmonary congestion and a failure of the right ventricle (Weymann et al., 2014b). Especially in the setting of a dilated, poorly contracting heart with severe systolic dysfunction, decompression by LV venting might be crucial for the recovery of the heart muscle.

In the majority of ECLS cases, the right ventricle is decompressed sufficiently by the venous cannula, placed at the cavo-atrial boundary. If LV venting is not applied the LV is only decompressed if a patent foramen ovale or a septum defect with left to right shunt is present. Furthermore, it has been described that in the setting of severe systolic dysfunction (low cardiac output) with ECLS in situ, that ECLS itself can cause distension of the left ventricle, increasing preload and reducing subendothelial perfusion impairing myocardial ischemia (Einzig et al., 1980). These patho-mechanisms can be avoided by unloading of the LV using a vent (Aiyagari et al., 2006; Weymann et al., 2014b). It has been demonstrated that LV-decompression by venting improves lung, heart and end organ recovery with the ability to wean successfully from ECLS or to bridge to ventricular assist device or even to heart transplantation (Sandrio et al., 2014).

Several case reports and small case series have described the potential for LV decompression to improve outcome of patients in cardiogenic shock (Aiyagari et al., 2006; Hacking et al., 2015; Koenig et al., 1993; Ward et al., 1995). Kotani et al. (2013) investigated one of the biggest cohorts of children treated by veno-arterial ECLS with left-heart decompression (LA venting) to improve LV function (23 of 178 patients). Kotani reports the importance of early initiating of decompression for the probability for successful weanig from ECLS. However, these reports only describe the management in paediatric patients or in very small cohorts and valid data about the outcome of adult patients with or without LV decompression has not been published yet. 
ECLS can be implanted either peripherally, usually by cannulation of the femoral, subclavian or axillary vessels, or centrally in combination with sternotomy. While peripheral ECLS (pECLS) by transcutaneously introducing venous and arterial cannulas into the target vessels is a simple and very fast technique, vessel injury, major bleeding and limb ischemia are common complications (Bisdas et al., 2011; Zimpfer et al., 2006). Today bypass techniques are established to reduce limb ischemia distal of the arterial cannulation. Peripheral ECLS with femoral vessel cannulation generates non-physiological retrograde arterial flow with the consequence that oxygenated blood may not reach coronary system or supra-aortic vessels (Secker-Walker et al., 1976). By comparison, cannulation of the axillary and subclavian artery is more time-consuming, requires (usually) a surgeon and vessels must be dissected carefully prior cannulation. In contrast to a fast peripheral approach, central ECLS (cECLS) therapy is more invasive and requires a surgical setting with thoracotomy. But cECLS generates a physiological antegrade flow support without the risk of peripheral vessel complications or under-perfusing organs. Although both techniques are highly standardized and logistics as well as equipment are available widely across specialized advanced heart failure units (Tschierschke, Katus \& Raake, 2013), insufficient recovery of the ventricles post-ECLS implantation remains a crucial complication of this therapy (Aiyagari et al., 2006).

The aim of this single centre study was to analyse the outcome of patients receiving peripheral and central ECLS and to evaluate whether decompression of the failing left ventricle by vent implantation has a positive influence on outcomes, with the view to optimize the therapeutic strategy in this complex patient cohort.

\section{MATERIALS AND METHODS}

\section{Patients}

\section{Study population}

Single-centre retrospective data was analysed of all patients with INTERMACS level 1 (cardiogenic shock with or without respiratory failure) who underwent ECLS implantation in our department between April 2004 and February 2014. The study design was approved by our institutional review board (Medical Faculty of the University Heidelberg, No. S-099/2015). Collectively, 48 adult patients with complete records could be identified (mean age $49.7 \pm 19.5$ years). Most common aetiology of cardiogenic shock was dilated cardiomyopathy ( $n=10,22.8 \%)$, followed by acute myocarditis $(n=9,18.8 \%)$ and acute myocardial infarction $(n=6,12.5 \%$, Table 1$)$. The majority received cECLS (with left ventricular decompression $n=20,41.7 \%$; without $n=18,37.5 \%$ ) whereas only $20.8 \%$ $(n=10)$ obtained pECLS. All pECLSs were implanted by cardiologists on the Cardiology Intensive Care unit (CCU) and none of the pECLS patients received a LV vent catheter. If patients were not in clinical stable conditions and therefore ground transportation not reasonable (departments are located in separate buildings without immediate connection) patients received pECLS on CCU. All patients treated at the Department of Cardiac Surgery received a cECLS. In addition, it became standard practice to add in left ventricular decompression in recent years. However, at the beginning of our study period individual 
Table 1 Aetiology of underlying disease.

\begin{tabular}{ll} 
Aetiology $(\boldsymbol{n = 4 8})$ & \\
\hline DCMP & $20.8 \%(n=10)$ \\
Acute myocarditis & $18.8 \%(n=9)$ \\
Myocardial infarction & $12.5 \%(n=6)$ \\
Aortic heart disease & $10.4 \%(n=5)$ \\
ICMP & $6.3 \%(n=3)$ \\
Others & $31.3 \%(n=15)$ \\
\hline
\end{tabular}

Notes.

Data are presented as percentage $(n)$. DCMP, dilated cardiomyopathy.

ICMP, ischemic cardiomyopathy.

surgeon preference determined whether a left ventricular decompression was applied or not. In our cohort, all LV vent catheters were implanted at time of initiation of ECLS.

The following clinical indicators for cardiogenic shock were applied ("Fast-EntryCriteria"): low cardiac index (CI) $(<2.2$ litres/min/m2), low systolic pressure $(<90 \mathrm{mmHg}$ for longer than $30 \mathrm{~min}$ ) and specific clinical indications of central and peripheral hypoperfusion (cold extremities, oliguria or altered mental condition), which are refractory to fluid substitution and intravenous inotropic support. We defined respiratory failure as acute hypoxemia, which is refractory to protective pulmonary ventilation $(\mathrm{PaO} 2<8.0 \mathrm{kPa}$, $\mathrm{PaCO} 2>6.7 \mathrm{kPa}, \mathrm{pH}<7.2$ at Fi02 1.00).

\section{Surgical procedures}

Implantation of cECLS was performed as previously described (Weymann et al., 2014b). Central ECLS implantation was established using a median sternotomy approach for the cannulation of the ascending aorta. A 22 French arterial cannula was inserted using the Seldinger technique (Edwards Lifesciences Corporation, Irvine, CA, USA). Venous return was established by cannulation of the right atrium using a 28 French cannula (Medtronic, Minneapolis, MN, USA) and by directing the tip of the cannula towards the tricuspid valve. In case of left ventricular decompression, a heparin-coated 24 French cannula was inserted via the right superior pulmonary vein into the left ventricle. The right atrial and the left ventricular cannula were connected together (Y-connector) to the inflow of the ECLS system. Furthermore, the ECLS consisted of a Thoratec ${ }^{\circledR}$ CentriMag blood pump (Thoratec ${ }^{\circledR}$ CentriMag ${ }^{\circledR}$ Blood Pump; Pleasanton, CA, USA) in combination with a D902 ECMO oxygenator (Dideco, Sorin Group, Milan, Italy). A detailed schematic illustration and postoperative picture of our cECLS setup is shown in Fig. 1.

We targeted an ECLS flow of $2.6 \mathrm{~L} / \mathrm{min} / \mathrm{m}^{2}$ body surface area. During the implantation procedure, transesophageal echocardiography (TEE) was performed routinely to obtain a correct position of all cannulas. Postoperatively, echocardiography was re-performed daily during intensive care unit (ICU) stay to ensure sufficient decompression of the left ventricle and to evaluate the ability of weaning. The LV decompression was always applied at the time of ECLS initiation.

In case of peripheral cannulation, cannulas were inserted transcutaneousely into the femoral artery (16 or 18 French OptiSite Arterial perfusion cannula, Edwards Life Science, 

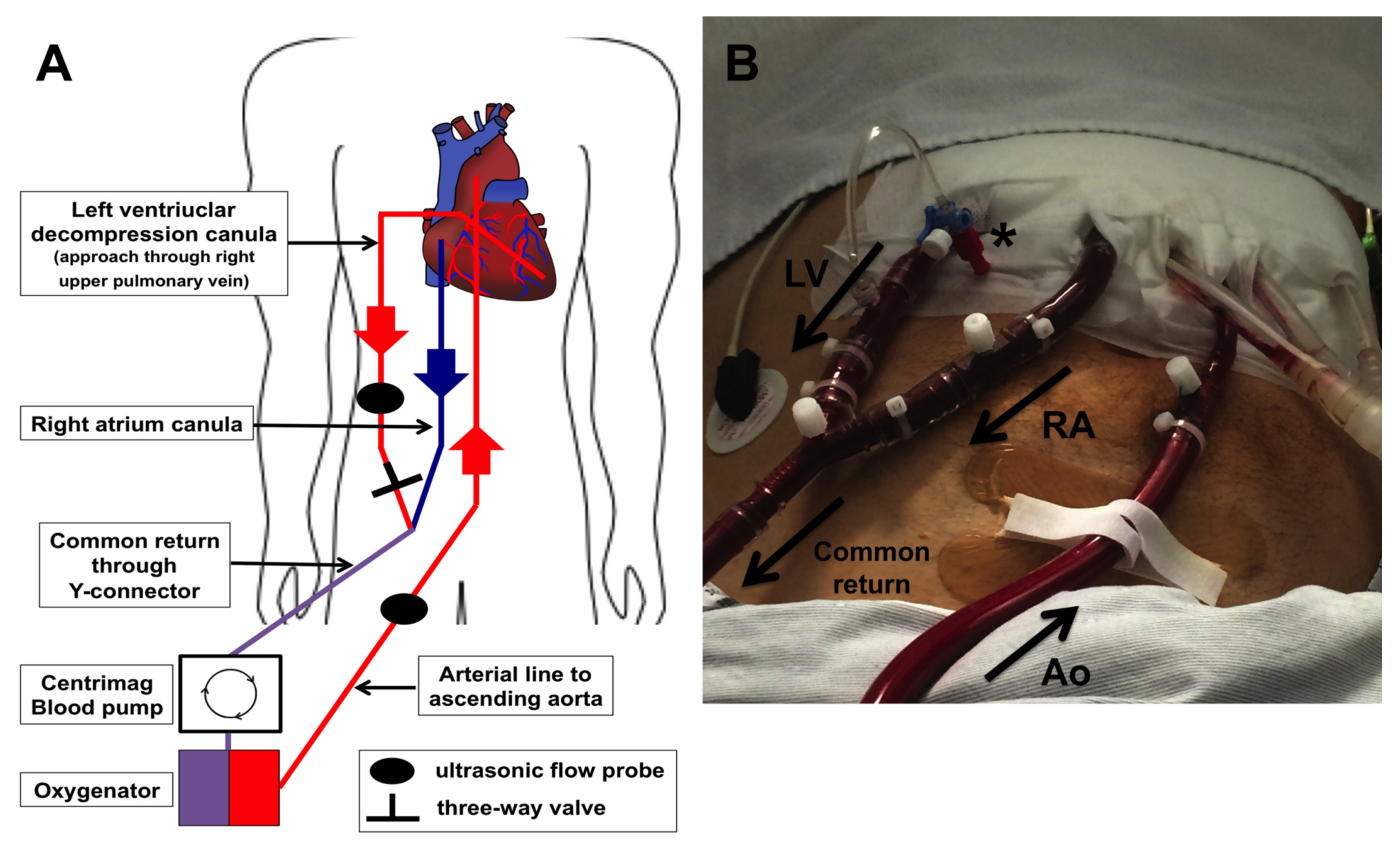

Figure 1 Illustration of central ECLS setup with LV vent (left ventricular). (A) Schematic illustration of cECLS (central extracorporeal life support) plus left ventricular decompression setup. (B) Postoperative picture showing ECLS assemblage on intensive care unit. LV decompression cannula (LV) is connected extracorporeal with the right atrial cannula (RA) to the common "venous" return of the circuit. A threeway valve is maintaining the ability to take blood gas samples of the LV blood to estimate lung perfusion as well as function. The aortic cannula (Ao) is implemented in the ascending aorta conducting the oxygenated blood back to the patient. Ultrasonic flow probes are applied on the outflow lines allowing flow metering.

Irvine CA, USA) and femoral vein (RAP femoral venous cannula 22 or 23/25 French; Sorin Group, Arvada, CO, USA) using the Seldinger technique. The position of the venous cannula (multi-drain cannula) was controlled by transesophageal echocardiography. Support was given by a portable centrifugal pump heart-lung support (Cardio Help; Maquet, Rastatt, Germany and Bio-Medicus ${ }^{\circledR}$; Medtronic, Meerbusch, Germany). Continuous monitoring of ECLS flow was secured by using ultrasonic probes (Transonics Inc.). Ideal oxygenation and decarboxylation was measured in short intervals using pointof-care blood gas measurement. In addition, continuous brain oximetry (near-infrared spectroscopy, INVOS; Covidien, Mansfield MA, USA) was monitored on ICU. By using the three-way-tap in the proximal venous line and the LV-decompression line, selective blood gas analyses from the left heart can detect left ventricular and pulmonary recovery. Increased blood flow of the left ventricular cannula with greater oxygen saturation enabled additional documentation of lung recovery.

\section{Weaning procedure}

Feasibility of weaning from ECLS was determined by evaluating the following: (i) possibility of intermittent reduction of the ECLS blood flow to a minimum of $1 / 3$ individual cardiac output (ii) preserved lung function (Horovitz Index $>200 \mathrm{~mm} \mathrm{Hg}$ ) with inspiration pressure at ventilator $<30 \mathrm{~cm} \mathrm{H}_{2} \mathrm{O}$ (iii) no signs of right ventricular failure (central venous 
pressure $<17 \mathrm{~cm} \mathrm{H}_{2} \mathrm{O}$ ) (iv) pulsatile systolic arterial blood pressure of $>90 \mathrm{~mm} \mathrm{Hg}$ under only low dosage of inotropic support for at least twelve hours (v) serum lactate levels $<3 \mathrm{mmol} / \mathrm{l}$ and (vi) central venous oxygen saturation $>65 \%$. If $L V$ vent was implanted, left atrial blood flow and gas analysis were evaluated.

\section{Statistics}

The study cohort was divided into two study groups: patients who underwent ECLS with LV venting and ECLS without LV venting. Furthermore, cECLS patient were divided into 2 subgroups, cECLS with and cECLS without LV venting for sub-analysis. Continuous variables are presented as mean \pm standard deviation or as median combined with range. Categorical data are shown as percentage. Differences in preoperative, operative and postoperative data were elaborated by Student's $t$ test and chi-square test. Survival function was calculated by use of Kaplan-Meier estimator and differences in survival were determined using log rank test. Multivariate logistic regression model (stepwise and backward likelihood ratio selection) was performed to determine the influence of vent support on 30-day survival. To adjust the estimated benefit by venting we selected a priori the covariates age and gender. Two-tailed probability values less than 0.05 were considered as significant and values less than 0.01 as highly significant. Statistical analysis was performed using common statistic software (IBM SPSS 22.0, Chicago, IL, USA).

\section{RESULTS}

\section{Baseline characterization and duration of support}

Follow up was completed successfully in 100\%, with a mean follow up of 269 days (standard deviation 629 days, maximum 2,828 days). We distinguished between vent vs. no vent group as well as between pECLS and cECLS with and without vent (sub-analysis, see supplemental content). We saw a homogeneous distribution of sex between the study groups. Patients with ECLS and LV-vent were significantly younger and had lower body mass index than patients without LV-vent (38.28 vs. 57.9 years; $p=0.001$ and 23.1 vs. 27.2 respectively, Table 2). Mean support on ECLS Patients was $6.10 \pm 3.81$ days. Length of ECLS support $(p=0.055)$, ventilation time $(p=0.091)$ and duration of in-hospital stay $(p=0.089) \mathrm{did}$ not differ significantly between the study groups (ECLS with vent vs. ECLS without vent). Patients of the non-venting group more often had intra-aortic balloon pump support before ECLS was implemented ( $p=0002$, Table 2$)$. Furthermore, the non-venting group contained more post-cardiotomy cases $(p=0.007)$.

Central ECLS plus LV vent was in use for significantly longer than cECLS without LV venting ( $7.35 \pm 4.16$ vs. $4,78 \pm 2.58$ days; $p=0.030$, see Table S2, supplemental content). Peripheral ECLS support was run for $6.0 \pm 4.47$ days, while there was no difference in support duration between pECLS and cECLS $(6,13 \pm 3.70$ days; $p=0.924)$.

\section{Development of clinical parameters in the course of ECLS support}

We evaluated liver, renal and lung function during extracorporeal life support. Specific points of time were: immediately prior to ECLS support, day one and day three on support or straight after ECLS explantation if weaning or bridging was possible. In our detailed 


\begin{tabular}{llll}
\hline Table 2 Baseline characteristic. & & & \\
Baseline variables $(\boldsymbol{n = 4 8 )}$ & w/ vent $(\boldsymbol{n}=\mathbf{2 0})$ & w/o vent $(\mathbf{2 8})$ & $\boldsymbol{p}$-value \\
\hline Female & $6(30)$ & $11(39)$ & 0.507 \\
Male & $14(70)$ & $9(50)$ & 0.208 \\
Age, years & $38.3 \pm 20.6$ & $57.9 \pm 14.0$ & $<0.01$ \\
BMI kg/m2 & $23.1 \pm 4.6$ & $27.2 \pm 4.5$ & 0.004 \\
Inotropic support preoperative & $20(100)$ & $18(100)$ & 1.000 \\
IABP pre ECLS & $2(10)$ & $15(54)$ & $<0.01$ \\
Portable heart lung support system & $7(35)$ & $2(11)$ & 0.015 \\
Mechanical ventilation, pre op & $14(70)$ & $9(50)$ & 0.208 \\
ECLS post cardiotomy & $2(10)$ & $13(46)$ & $<0.01$ \\
\hline
\end{tabular}

Notes.

Data are presented as $n$ (percentage) or mean \pm standard deviation (SD). Student $t$ test for continuous variables or Chisquared test for categorical variables. A probability value ( $p$-value) of $<0.05$ was considered significant.

BMI, Body Mass Index; ECLS, extracorporeal life support; IABP, intra-aortic balloon pump; w/, with; w/o, without..

analysis, we could not find relevant differences in end-organ function parameters between the groups vent vs. no vent. Sub-analysis revealed, that pre-implantation assessed Horovitz index was higher in the cECLS vent group compared to no vent group ( $214.21 \pm 128.39$ vs. $109.38 \pm 63.04, p=0.019$, see Table S4, supplemental content), whereas at the same time the number of patients on respirator support was higher within this group. However, by definition, the majority of the patients suffered from a respiratory distress syndrome with a Horovitz index less than $200 \mathrm{mmHg}$ (ARDS Definition Task Force et al., 2012).

\section{ECLS as therapeutic bridge}

Four patients (8.3\%) were successfully bridged to recovery by cECLS (one with vent and 3 without vent). The majority of survivors were bridged to VAD Systems (ventricular assist device, $n=14,29.2 \%)$, whereas $6(12.5 \%)$ patients underwent heart transplantation over the course of time ( 5 patients were bridged to transplant and 1 patients was bridged to VAD and then bridged to transplant, Table 3). Patients with LV-venting were more frequently bridged to VAD (bridge to bridge, $p<0.01$, Table 3). Moreover, mortality rate under ECLS support was higher in patients without LV venting $(p=0.027)$. Sub-analysis revealed, that patients with cECLS and additional vent implantation were more frequently bridged to a VAD system than patients with cECLS without venting $(50.0 \%$ vs. $5.6 \%, p \leq 0.01)$.

\section{Left ventricular venting improves short-term survival}

Beyond organ function and clinical parameters, the principal purpose of ECLS in cardiogenic shock is the improvement of survival. Overall thirty-day survival was $37.5 \%$ $(n=18)$ in the overall study population. Patients with LV decompression by venting had a superior short-term survival after 30 -days (55\% vs. $25 \%, p=0.034$,) but no favourable survival after 6 and 12 months ( $p=0.110$ and 0.198 respectively, Table 3 ). Concerning long-term survival by Kaplan-Meier estimation, patients with LV vent showed a trend but no significant superiority (log-rank test $p=0.066$, Fig. $2 \mathrm{~A}$ ) compared to patients without LV decompression. In addition, long-term survival did not differ between subgroups pECLS, cECLS without and cECLS with vent ( $p=0.183$, Fig. $2 \mathrm{~B})$. 
Table 3 Outcome variables.

\begin{tabular}{llll} 
Outcome variables $(\boldsymbol{n = 4 8 )}$ & w/ vent $(\boldsymbol{n = 2 0 )}$ & w/o vent $(\mathbf{2 8})$ & $\boldsymbol{p}$-value \\
\hline Length of support, days & $7.4 \pm 4.2$ & $5.2 \pm 3.4$ & 0.055 \\
Hospital stay, days & $54.2 \pm 62.71$ & $28.9 \pm 34.9$ & 0.089 \\
Mechanical ventilation, hours & $761.3 \pm 1,047.8$ & $383.5 \pm 426.4$ & 0.091 \\
Bridge to recovery & $1(5)$ & $3(11)$ & 0.442 \\
Bridge to transplant & $4(20)$ & $1(4)$ & 0.146 \\
Bridge to bridge & $10(50)$ & $4(14)$ & $<0.01$ \\
Exitus during support & $5(25)$ & $16(57)$ & 0.027 \\
30-day survival & $11(55)$ & $7(25)$ & 0.034 \\
6-month survival & $8(40)$ & $5(18)$ & 0.110 \\
1-year survival & $7(35)$ & $5(18)$ & 0.198 \\
ECLS post cardiotomy & $2(10)$ & $13(46)$ & $<0.01$ \\
\hline
\end{tabular}

Notes.

Data are presented as $n$ (percentage) Chi-squared test for categorical variables. A probability value ( $p$-value) of $<0.05$ was considered significant.

ECLS, extracorporeal life support; w/, with; w/o, without.

Table 4 Multivariate logistic regression model for 30-day mortality.

\begin{tabular}{lll} 
Variable & $\boldsymbol{p}$-value & OR $(\mathbf{9 5} \% \mathbf{C I})$ \\
\hline ECLS w/o vent & 0.038 & $3.667(1.074-12.518)$ \\
Age & 0.089 & $1.028(0.996-1.016)$ \\
Male gender & 0.815 & $0.864(0.253-2.953)$ \\
\hline
\end{tabular}

Notes.

Multivariate logistic regression model for analysis of 30-day mortality adjusted for age and gender. Stepwise and backward likelihood ratio selection. A probability value ( $p$-value) of $<0.05$ was considered as significant.

CI, confidence interval; ECLS w/o vent, extracorporeal life support without venting; OR, Odds ratio.

\section{No-vent support as independent risk factors for 30-day mortality}

Multivariate logistic regression model adjusted for age and gender revealed ECLS without vent as an independent risk factor for 30-day mortality (Odds ratio 3.667, 95\% CI [1.07412.518], $p=0.038$, Table 4).

\section{DISCUSSION}

Independent of the underlying disease, acute therapy of cardiogenic shock remains a challenge for all disciplines involved. Taking into account, that conservatively treated cardiogenic shock is associated with a tremendously high mortality, ECLS therapy, if available, remains often the last chance for recovery (Mohite et al., 2014). ECLS utilization has increased in recent years, monitored by the ECLS Registry group, showing an acceptable overall survival of 44\% (Paden et al., 2013). Our data represent nearly identical over all with a 30 -day survival of $37.5 \%$. Altogether, survival of patients in cardiogenic shock has improved significantly over the last decades, mainly due to advances in medical treatment, revascularization techniques, intensive care and mechanical support. Goldberg et al. (2009) reported improving trends in the hospital prognosis of patients with acute myocardial 
A

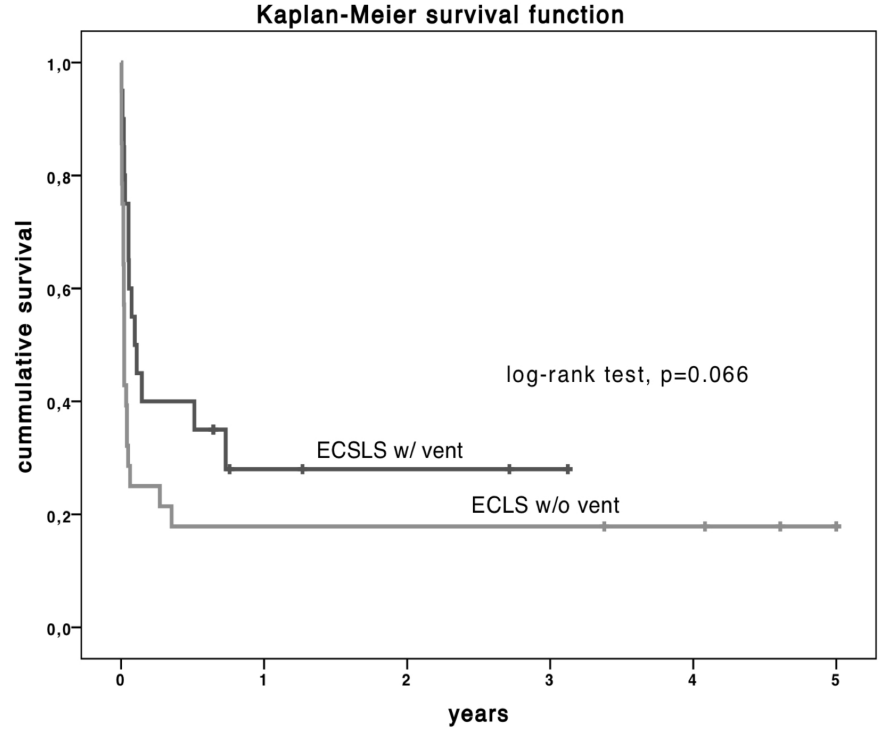

number of patients at risk

$\begin{array}{llllllll}\text { ECLS w/ vent } & 20 & 8 & 3 & 2 & 1 & 0 & 0 \\ \text { ECLS w/o vent } & 28 & 4 & 4 & 4 & 4 & 3 & 1\end{array}$

B

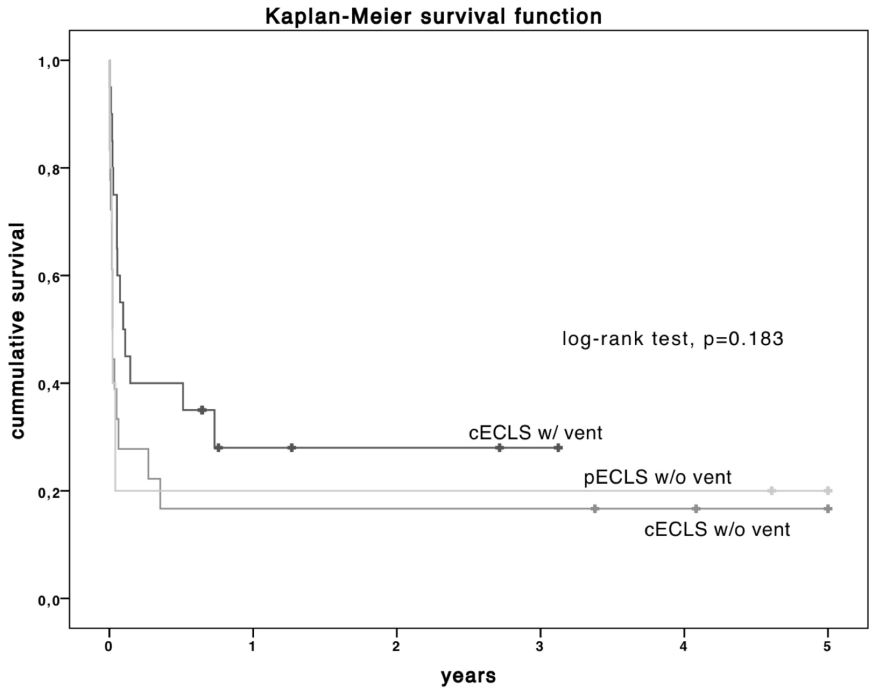

number of patients at risk

$\begin{array}{llllllll}\text { cECLS w/vent } & 20 & 8 & 3 & 2 & 1 & 0 & 0 \\ \text { pECLS w/o vent } & 10 & 2 & 2 & 2 & 2 & 2 & 0 \\ \text { cECLS w/o vent } & 18 & 3 & 3 & 3 & 3 & 2 & 0\end{array}$

Figure 2 Estimated Kaplan-Meyer (KM) survival function (log-rank test) of the total cohort diversified by vent implantation (A) and by type of ECLS support (B) over 5-year follow up. cECLS w/ vent vs. cECLS w/o vent, $p=0.102$; pECLS w/o vent vs. cECLS w/ vent, $p=0.117$ and $p E C L S$ w/o vent vs. cECLS w/o vent, $p=0.950$ and peripheral ECLS vs. central ECLS, $p=0.345$. cECLS, central extracorporeal life support; pECLS, peripheral extracorporeal life support; w/, with; w/o, without. 
infarction and associated cardiogenic shock in a large three-decade-long retrospective study (from 1975 to 2005) with 13,663 patients.

In general, ECLS therapy without LV decompression is mainly limited by lung failure (pulmonary oedema with impaired oxygenation) and unrecognized left ventricular distension resulting in ventricular pressure overload, restricted coronary blood flow and insufficient cardiac recovery. The importance of left side decompression in ECLS has been described before. However, these publications report the use of balloons, blades or stentings to achieve an interatrial left to right side shunt (Haynes et al., 2009; Seib et al., 1999). Aiyagari et al. (2006) published their promising experience within a small cohort treated with a selective trans-septal left atrial cannulation to drain blood into the ECLS circuit. When evaluating mortality rates, death after cardiogenic shock occurs early even if ECLS support is given. In our cohort, $62.5 \%$ ( $n=30$ out of 41 decedents after one year) did not survive 30 days after ECLS implantation. Patients suffering from cardiogenic shock are by definition critically unwell and haemodynamically decompensated, with subsequently lung and end-organ dysfunction leading to fatal multi-organ failure.

End-organ dysfunction is the result of three obvious risk factors. The first is hypoxemia, itself a result of lung failure due to an impediment of the failing LV (or isolated/combined right heart failure), causing destruction and apoptosis of parenchymal cells. Secondly ischemia, due to reduced organ perfusion and finally congestion due to increased venous backflow.

Clinical parameters such as renal and liver function as well as parameters describing the lung function all failed to explain the beneficial outcome.

An important advantage of ECLS is the ability to instantly and continuously measure the blood flow crossing the lungs by Doppler-ultrasound measurement and pO2 of the LV decompressing cannula is of vital importance in terms of evaluation and timing of a potential weaning from ECLS or bridging to a further long-term support (e.g., VAD or transplant). These sophisticated options for heart and lung function evaluation allowed a more accurate timing to wean and are an important key to keep ECLS support duration as short as possible, but as long as required.

The optimal approach for utilizing ECLS remains debatable. The central approach via sternotomy allows a fast and excellent access to both right and left atrium as well as the ascending aorta. However, the surgical trauma is immense and early postoperative mobilization is limited. By comparison, peripheral support is of growing interest, due to a simple approach and a pervasively available technique in intensive care units without the need of immediate surgical support (Basra, Loyalka \& Kar, 2011; Kar et al., 2012; Kar et al., 2011). At present, peripheral support is associated with complications like limb ischemia (Bisdas et al., 2011; Foley et al., 2010) and insufficient supply of oxygenated blood to both, the heart and the brain (Bisdas et al., 2011; Secker-Walker et al., 1976; Werdan et al., 2014). Moreover, retrograde flow is associated with a greater risk of aortic root thrombosis possibly resulting in cerebral insult and/or peripheral embolism, especially if no ejection is provided by the LV itself (Leontiadis et al., 2010) and an impediment of the weaning procedure due to the counter flow if support is reduced. However, if peripheral support is favoured or central support is not possible because of missing resources or logistics, we suggest setting 
up a transcutaneous left side decompression as well. Therefore, LV decompression could be accomplished by placing a transvenous cannula crossing the interatrial septum into the left atrium and connected to the venous return as previously described. Other groups have described the use of a trans-aortic decompression to unload the left ventricle in addition to a veno-arterial ECLS (Beurtheret et al., 2012; Koeckert et al., 2011). We are not in favour of additional trans-aortic decompression as first line procedure, mainly due to one very important limitation of this setting (if pECLS is implanted through the femoral vessels). The antegrade flow of the trans-aortic decompression catheter (usually implanted through the femoral artery) and the retrograde flow of the pECLS system are opposing and create non-predictable organ perfusion. In worst case this setting might even create a state of no-flow with need for increased vasopressor support. Moreover, while not important clinically, the costs of using two devices is significantly higher.

\section{LV vent should be applied to improve ECLS outcome}

Our analysis demonstrated that ECLS without venting has a significantly inferior outcome after 30 days compared to ECLS with venting. Furthermore, mortality rate was significantly lower in patients with LV venting during ECLS support and non-ventilatory support was determined as strong independent risk factor for 30-day mortality. Even though our sub-analysis of patients with cECLS did not reveal a definite survival benefit for LV venting, we propose a combination of cECLS approach with LV decompression to justify the increase of invasiveness compared to the simpler pECLS approach. Moreover, a de-novo sternotomy may be avoided by using alternative approaches. These techniques have been described before by Weymann et al. (2014a) reporting a minimal invasive technique and avoiding sternotomy by approaching the heart via left lateral thoracotomy. Using the lateral entry, an additional LV decompression cannula can be added easily and tunnelled as described. Combination of both procedures provides the advantages of central support, LV decompression and the benefits of avoiding sternotomy, allowing early extubation and mobilization. In order to reduce surgical trauma and to make surgical procedure more feasible the LVAD outflow graft can be anastomosed to cannulate the aortic line, enabling an easy switch to long-term support (Weymann, Simon \& Popov, 2014c).

Within our department, the vast majority of central ECLS procedures were performed using a LV vent within recent years, as a consequence of our growing experience with ECLS. Furthermore, the favourable results of LV-venting may be biased or confounded by generally improved perioperative management. This includes the continuous and systematic development of surgical strategies as well as the more sophisticated management on intensive care unit prior and post ECLS implantation.

\section{Left ventricular venting in pECLS?}

As presented in our data cECLS alone did not lead to improved outcomes compared to pECLS. In our opinion, LV-venting must be performed to justify the much more invasive cECLS procedure. However, at time of writing there are no large studies available presenting pECLS with LV decompression and comparing different strategies. If a pECLS is implemented, LV-decompression could also be achieved by draining left atrial volume via a 
trans-septal cannula by a transaortic Impella ${ }^{\circledR}$ system. A randomized multi-center study is required to address these essential major questions on this topic: (i) Is a routine peripheral support a non-inferior option to maintain ECLS compared to the central approach? (ii) Which technique (and/or device application) of LV decompression is best in pECLS, in a scenario where the central approach is not favoured or applicable?

\section{CONCLUSION}

ECLS is a powerful and oftentimes end-stage tool to overcome life threatening cardiogenic shock, regardless of the underlying disease. We were able to demonstrate the benefit of LV venting in terms of 30-day survival, the most critical time frame in the state of refractory cardiogenic shock, independently of underlying cause. If ECLS is implemented, we strongly recommend this be done alongside decompression of the left ventricle. The technique of a separate LV-decompression in cECLS allows sophisticated options to follow both, cardiac and pulmonary recovery. Cardiogenic shock remains a highly challenging condition to manage and morbidity and mortality outcomes remain poor. To improve survival and to allow for further treatment options, using all available resources including therapy-enhancing techniques like LV-decompression are a vital next step that require further data to support and qualify their use and optimisation.

\section{Limitations}

Our study demonstrates a relatively high number of ECLS patients with a specific LV decompression technique and a complete follow up. However, this study is limited by the fact that we performed a retrospective single-center analysis containing patients with a large variability of underlying diseases causing cardiogenic shock. Furthermore, all pECLS were implanted by Cardiologist on the Cardiology Care Unit and no LV venting was applied in pECLS patients, which leads to a certain bias.

\section{ACKNOWLEDGEMENTS}

The authors thank Joel Schamroth MD, for providing an excellent scientific language editing.

\section{ADDITIONAL INFORMATION AND DECLARATIONS}

\section{Funding}

This work was supported by the Deutsche Forschungsgemeinschaft (DFG) and RuprechtKarls-Universität Heidelberg within the funding programme Open Access Publishing. The funders had no role in study design, data collection and analysis, decision to publish, or preparation of the manuscript.

\section{Grant Disclosures}

The following grant information was disclosed by the authors:

Deutsche Forschungsgemeinschaft (DFG).

Ruprecht-Karls-Universität Heidelberg. 


\section{Competing Interests}

The authors have no conflicts of interest to declare. The authors declare that the co-author AM, Associate Professor, is a member of the Advisory Board and Editors of PeerJ (Section Emergency \& Critical Care). This does not alter the authors' adherence to all of the PeerJ's policies.

\section{Author Contributions}

- Bastian Schmack, Philipp Seppelt and Arjang Ruhparwar conceived and designed the experiments, performed the experiments, analyzed the data, contributed reagents/materials/analysis tools, wrote the paper, prepared figures and/or tables, reviewed drafts of the paper, conception.

- Alexander Weymann conceived and designed the experiments, performed the experiments, analyzed the data, contributed reagents/materials/analysis tools, reviewed drafts of the paper, conception.

- Christina Alt performed the experiments, analyzed the data, contributed reagents/materials/analysis tools, wrote the paper, prepared figures and/or tables, reviewed drafts of the paper.

- Mina Farag, Rawa Arif and Klaus Kallenbach performed the experiments, analyzed the data, reviewed drafts of the paper.

- Andreas O. Doesch, Philip W. Raake and Aron-Frederik Popov analyzed the data, reviewed drafts of the paper.

- Ashham Mansur analyzed the data, wrote the paper, reviewed drafts of the paper.

- Matthias Karck performed the experiments, analyzed the data, wrote the paper, reviewed drafts of the paper, conception.

\section{Human Ethics}

The following information was supplied relating to ethical approvals (i.e., approving body and any reference numbers):

The study design was approved by the institutional review board of the Medical Faculty of the University Heidelberg (No. S-099/2015).

\section{Data Availability}

The following information was supplied regarding data availability:

The raw data has been uploaded as a Supplemental File.

\section{Supplemental Information}

Supplemental information for this article can be found online at http://dx.doi.org/10.7717/ peerj.3813\#supplemental-information.

\section{REFERENCES}

Aiyagari RM, Rocchini AP, Remenapp RT, Graziano JN. 2006. Decompression of the left atrium during extracorporeal membrane oxygenation using a transseptal cannula incorporated into the circuit. Critical Care Medicine 34:2603-2606 DOI 10.1097/01.CCM.0000239113.02836.F1. 
ARDS Definition Task Force, Ranieri VM, Rubenfeld GD, Thompson BT, Ferguson

ND, Caldwell E, Fan E, Camporota L, Slutsky AS. 2012. Acute respiratory distress syndrome: the Berlin Definition. Journal of the American Medical Association 307:2526-2533 DOI 10.1001/jama.2012.5669.

Awad HH, Anderson Jr FA, Gore JM, Goodman SG, Goldberg RJ. 2012. Cardiogenic shock complicating acute coronary syndromes: insights from the Global Registry of Acute Coronary Events. American Heart Journal 163:963-971 DOI 10.1016/j.ahj.2012.03.003.

Basra SS, Loyalka P, Kar B. 2011. Current status of percutaneous ventricular assist devices for cardiogenic shock. Current Opinion in Cardiology 26:548-554 DOI 10.1097/HCO.0b013e32834b803c.

Beurtheret S, Mordant P, Pavie A, Leprince P. 2012. Impella and extracorporeal membrane oxygenation: a demanding combination. ASAIO Journal 58:291-293 DOI 10.1097/MAT.0b013e31824c38ef.

Bisdas T, Beutel G, Warnecke G, Hoeper MM, Kuehn C, Haverich A, Teebken OE. 2011. Vascular complications in patients undergoing femoral cannulation for extracorporeal membrane oxygenation support. Annals of Thoracic Surgery 92:626-631 DOI 10.1016/j.athoracsur.2011.02.018.

Combes A, Leprince P, Luyt CE, Bonnet N, Trouillet JL, Leger P, Pavie A, Chastre J. 2008. Outcomes and long-term quality-of-life of patients supported by extracorporeal membrane oxygenation for refractory cardiogenic shock. Critical Care Medicine 36:1404-1411 DOI 10.1097/CCM.0b013e31816f7cf7.

Einzig S, Staley NA, Mettler E, Nicoloff DM, Noren GR. 1980. Regional myocardial blood flow and cardiac function in a naturally occurring congestive cardiomyopathy of turkeys. Cardiovascular Research 14:396-407 DOI 10.1093/cvr/14.7.396.

Foley PJ, Morris RJ, Woo EY, Acker MA, Wang GJ, Fairman RM, Jackson BM. 2010. Limb ischemia during femoral cannulation for cardiopulmonary support. Journal of Vascular Surgery 52:850-853 DOI 10.1016/j.jvs.2010.05.012.

Goldberg RJ, Spencer FA, Gore JM, Lessard D, Yarzebski J. 2009. Thirty-year trends (1975 to 2005) in the magnitude of, management of, and hospital death rates associated with cardiogenic shock in patients with acute myocardial infarction: a population-based perspective. Circulation 119:1211-1219 DOI 10.1161/CIRCULATIONAHA.108.814947.

Hacking DF, Best D, D'Udekem Y, Brizard CP, Konstantinov IE, Millar J, Butt W. 2015. Elective decompression of the left ventricle in pediatric patients may reduce the duration of venoarterial extracorporeal membrane oxygenation. Artificial Organs 39:319-326 DOI 10.1111/aor.12390.

Haynes S, Kerber RE, Johnson FL, Lynch WR, Divekar A. 2009. Left heart decompression by atrial stenting during extracorporeal membrane oxygenation. International Journal of Artificial Organs 32:240-242.

Hochman JS, Boland J, Sleeper LA, Porway M, Brinker J, Col J, Jacobs A, Slater J, Miller D, Wasserman H, Menegus MA, David Talley J, McKinlay S, Sanborn T, LeJemtel T. 1995. Current spectrum of cardiogenic shock and effect of early revascularization 
on mortality. Results of an International Registry. SHOCK Registry Investigators. Circulation 91:873-881 DOI 10.1161/01.CIR.91.3.873.

Kar B, Basra SS, Shah NR, Loyalka P. 2012. Percutaneous circulatory support in cardiogenic shock: interventional bridge to recovery. Circulation 125:1809-1817 DOI 10.1161/CIRCULATIONAHA.111.040220.

Kar B, Gregoric ID, Basra SS, Idelchik GM, Loyalka P. 2011. The percutaneous ventricular assist device in severe refractory cardiogenic shock. Journal of the American College of Cardiology 57:688-696 DOI 10.1016/j.jacc.2010.08.613.

Koeckert MS, Jorde UP, Naka Y, Moses JW, Takayama H. 2011. Impella LP 2.5 for left ventricular unloading during venoarterial extracorporeal membrane oxygenation support. Journal of Cardiac Surgery 26:666-668

DOI 10.1111/j.1540-8191.2011.01338.x.

Koenig PR, Ralston MA, Kimball TR, Meyer RA, Daniels SR, Schwartz DC. 1993. Balloon atrial septostomy for left ventricular decompression in patients receiving extracorporeal membrane oxygenation for myocardial failure. Jornal de Pediatria 122:S95-S99.

Kotani Y, Chetan D, Rodrigues W, Sivarajan VB, Gruenwald C, Guerguerian AM, Van Arsdell GS, Honjo O. 2013. Left atrial decompression during venoarterial extracorporeal membrane oxygenation for left ventricular failure in children: current strategy and clinical outcomes. Artificial Organs 37:29-36 DOI 10.1111/j.1525-1594.2012.01534.x.

Leontiadis E, Koertke H, Bairaktaris A, Koerfer R. 2010. Thrombosis of the ascending aorta during mechanical circulatory support in a patient with cardiogenic shock. Interactive Cardiovascular and Thoracic Surgery 11:510-511 DOI 10.1510/icvts.2010.240689.

Mohite PN, Zych B, Banner NR, Simon AR. 2014. Refractory heart failure dependent on short-term mechanical circulatory support: what next? Heart transplant or long-term ventricular assist device. Artificial Organs 38:276-281 DOI 10.1111/aor.12157.

Paden ML, Conrad SA, Rycus PT, Thiagarajan RR, Registry E. 2013. Extracorporeal life support organization registry report 2012. ASAIO Journal 59:202-210 DOI 10.1097/MAT.0b013e3182904a52.

Sandrio S, Springer W, Karck M, Gorenflo M, Weymann A, Ruhparwar A, Loukanov T. 2014. Extracorporeal life support with an integrated left ventricular vent in children with a low cardiac output. Cardiology in the Young 24:654-660 DOI 10.1017/S1047951113001017.

Secker-Walker JS, Edmonds JF, Spratt EH, Conn AW. 1976. The source of coronary perfusion during partial bypass for extracorporeal membrane oxygenation (ECMO). Annals of Thoracic Surgery 21:138-143 DOI 10.1016/S0003-4975(10)64277-4.

Seib PM, Faulkner SC, Erickson CC, Van Devanter SH, Harrell JE, Fasules JW, Frazier EA, Morrow WR. 1999. Blade and balloon atrial septostomy for left heart decompression in patients with severe ventricular dysfunction on extracorporeal membrane oxygenation. Catheterization and Cardiovascular Interventions 46(2):179-186. 
TRIUMPH Investigators, Alexander JH, Reynolds HR, Stebbins AL, Dzavik V, Harrington RA, Van de Werf F, Hochman JS. 2007. Effect of tilarginine acetate in patients with acute myocardial infarction and cardiogenic shock: the TRIUMPH randomized controlled trial. Journal of the American Medical Association 297:1657-1666 DOI 10.1001/jama.297.15.joc70035.

Tschierschke R, Katus HA, Raake PW. 2013. First "Advanced Heart Failure Unit" at the Heart Centre of the University hospital Heidelberg. Deutsche Medizinische Wochenschrift 138:603-607 DOI 10.1055/s-0032-1332996.

Ward KE, Tuggle DW, Gessouroun MR, Overholt ED, Mantor PC. 1995. Transseptal decompression of the left heart during ECMO for severe myocarditis. Annals of Thoracic Surgery 59:749-751 DOI 10.1016/0003-4975(94)00579-6.

Werdan K, Gielen S, Ebelt H, Hochman JS. 2014. Mechanical circulatory support in cardiogenic shock. European Heart Journal 35:156-167 DOI 10.1093/eurheartj/eht248.

Weymann A, Sabashnikov A, Patil NP, Mohite PN, Zych B, Garcia Saez D, Popov AF, Simon AR. 2014a. Minimally invasive access for central extracorporeal life support: how we do it. Artificial Organs 39(2):179-181 DOI 10.1111/aor.12344.

Weymann A, Schmack B, Sabashnikov A, Bowles CT, Raake P, Arif R, Verch M, Tochtermann U, Roggenbach J, Popov AF, Simon AR, Karck M, Ruhparwar A. 2014b. Central extracorporeal life support with left ventricular decompression for the treatment of refractory cardiogenic shock and lung failure. Journal of Cardiac Surgery 9:60 DOI 10.1186/1749-8090-9-60.

Weymann A, Simon AR, Popov AF. 2014c. Modified extracorporeal life support: the Harefield technique. Asian Cardiovascular and Thoracic Annals 24(3):289 DOI 10.1177/0218492314552299.

Zimpfer D, Heinisch B, Czerny M, Hoelzenbein T, Taghavi S, Wolner E, Grimm M. 2006. Late vascular complications after extracorporeal membrane oxygenation support. Annals of Thoracic Surgery 81:892-895 DOI 10.1016/j.athoracsur.2005.09.066. 